
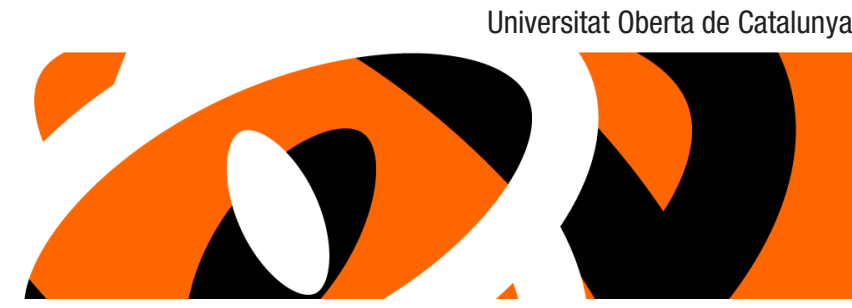

http://artnodes.uoc.edu

\title{
Investigación en diseño: suturando cuerpos, cacharros, epistemologías y lunas
}

\author{
Jaron Rowan \\ Marta Camps \\ BAU \\ Centro Universitario de Diseño de Barcelona
}

Fecha de recepción: agosto de 2017

Fecha de aceptación: octubre de 2017

Fecha de publicación: diciembre 2017

\section{Resumen}

La pregunta en torno a qué constituye investigación en arte y diseño está de plena vigencia y no son pocas las personas ni pocas las escuelas en las que se ha abordado esta cuestión anteriormente. En el siguiente artículo nos interesa abordar una perspectiva que, por lo general, está ausente de muchos de estos debates y que consideramos que es importante tener en cuenta: cómo pensar esta realidad sin tener que escindir el diseño de lo político, de lo social, de lo ético o de lo estético. Es decir, cómo pensar la investigación en diseño como una práctica situada, con sus materialidades específicas y lenguajes de investigación. Partiendo del trabajo de autores como Bruno Latour, Isabelle Stengers o Viveiros de Castro, vamos a proponer una genealogía truncada de la investigación en diseño que consideramos tiene el potencial de superar algunas de las escisiones epistemológicas que nos ha dejado la modernidad, que estableció falsas dicotomías entre hacer y pensar, conocer y sentir, investigar y jugar, razonar e intuir.

\section{Palabras clave}

investigación en diseño, epistemologías raras, pedagogía, modernidad

\section{Design research: suturing bodies, puppies, epistemologies and moons}

\begin{abstract}
The question around which this research into art and design is structured is highly topical. More than a few people and colleges have previously tackled this issue. In the following article, we aim to take a perspective that is generally overlooked in many of these debates and which, in our opinion, is important to take into account: how can we think about this reality without having
\end{abstract}




\section{artnodes}

http://artnodes.uoc.edu

to separate design from the political, social, ethical and aesthetic. In other words, how can we think about design research as a situated practice, with its specific research materialities and languages. Taking the work of authors such as Bruno Latour, Isabelle Stengers and Viveiros de Castro as a starting point, we are going to propose a truncated genealogy of design research that we believe has the potential to overcome some of the epistemological schisms that we have been left by modernity, which established false dichotomies between doing and thinking, knowing and feeling, investigating and playing, reasoning and sensing.

\section{Keywords}

design research, unusual epistemologies, pedagogy, modernity

\section{Introducción: el amanecer}

\author{
Yo he visto las estrellas. \\ ¿Qué son estrellas?, dicen \\ las hormigas inquietas. \\ Y el caracol pregunta \\ pensativo: ¿estrellas? \\ Sí, repite la hormiga. \\ He visto las estrellas. \\ Subí al árbol más alto \\ que tiene la alameda \\ y vi miles de ojos \\ dentro de mis tinieblas.
}

«Los encuentros de un caracol aventurero», Federico García Lorca

La pregunta en torno a qué constituye investigación en arte y diseño no es nueva, pero, sin duda, está vigente. No son pocas las personas ni las escuelas que han abordado esta cuestión con anterioridad. Quizás por eso, en este contexto de crisis y de incertidumbre, resulta tan interesante poder contribuir a este debate, centrándonos de forma específica en el diseño contemporáneo y su capacidad para devenir un espacio epistémico. Por motivos de espacio y dada nuestra intención de proponer una nueva mirada sobre el fenómeno, a continuación no vamos a revisar todas las diferentes tradiciones y obras que ya han abordado este fenómeno, que sabemos que son abundantes. Este problema ha asumido diferentes encarnaciones en función del contexto geográfico, de la época o del espacio o de la disciplina en el que aconteciera. En el Estado español, por ejemplo, ha estado siempre más cercano al ámbito de reflexión incluido bajo las siglas ACYT (Arte, Ciencia y Tecnología), mientras en el Reino Unido se ha abordado más desde lo que se denomina practice based research; ${ }^{1}$ es decir, la preocupación ha radicado en comprender cómo se pueden incorporar formas de investigación basadas en prácticas artísticas
0 creativas específicas. De forma más reciente, los denominados Estudios sociales de la ciencia y la tecnología, desde una perspectiva mucho más teórica, han mostrado también un interés por entender la investigación en diseño (Farias y Wilkie, 2015; Sánchez Criado y Rodríguez-Giralt, 2016), atreviéndose a incorporar en ocasiones cacharros y artefactos de diseño, como dispositivos capaces de recabar formas de información que las etnografías tradicionales parecían ignorar.

Lamentablemente, estas diferentes corrientes, pese a constituir un campo rico de aportaciones, no confluyen en una visión coherente 0 articulada en torno a qué constituye la investigación en diseño. $A$ esta proliferación de disciplinas habría que añadir el propio espacio del diseño, que de forma reciente ha puesto esta pregunta en un lugar central de sus prácticas. Desde que Christopher Frayling, ya en su famoso artículo «Research in Art and Design» (1994), distinguiera y categorizara la investigación en, para y a través del diseño, no son pocos los lugares en los que se ha profundizado y se ha continuado debatiendo en torno a este problema (Dunne y Raby, 2013; Markussen y Steino, 2012). A continuación, sin pretender dejar de lado esta extensa bibliografía nos interesa abordar una perspectiva que, por lo general, consideramos que está ausente de muchos de los debates en torno a la investigación en diseño y que es importante tener en cuenta: cómo pensar esta realidad sin tener que escindir el diseño de lo político, de lo social, de lo ético o de lo estético. Es decir, cómo pensar la investigación en diseño como una práctica situada, con sus materialidades específicas y lenguajes de investigación. Pero, primero, vamos a mirar la luna.

\section{Luna menguante}

El filósofo y antropólogo francés Bruno Latour relata la importancia que tuvo para Galileo haber sido, además de un hombre letrado en las ciencias, una persona interesada por el dibujo. Para este autor, si

1. Investigación basada en prácticas o proyectos. 


\section{artnodes}

http://artnodes.uoc.edu

Galileo fue capaz de entender la Luna a través de su telescopio no fue tan solo por tener a su disposición un instrumento óptico que otros ya habían usado con el mismo fin, sino por tener la mirada entrenada para ver juegos de luces y sombras. En sus propias palabras: «Si Galileo no hubiera sido un dibujante bueno (ya alguien acostumbrado a seguir las leyes de la perspectiva recientemente descubiertas), nunca hubiera interpretado las sombras que veía sobre la Luna como la proyección de las montañas; en cierto sentido, no habría "visto" nada en su lente, salvo unas manchas tornasoladas» (Latour, 2000). Su práctica como dibujante le había hecho sensible a cosas que otras personas podían mirar, pero no por ello ver. Desde aquí nos interesa explorar esta idea, la investigación entendida como una forma de hacerse sensible. Como un conjunto de espacios y prácticas que te permiten experimentar, sentir y ver las cosas de forma diferente.

Galileo no tan solo miró la Luna, la vio, o al menos vio la Luna que todas y todos hemos aprendido a ver. Es un buen ejemplo sobre cómo investigar tiene que ver con permitir que diferentes saberes conjuguen, entren en contacto. La intersección de diferentes tipos de saberes y prácticas permiten a Galileo hacerse sensible a la Luna. Pese a la importancia de este hecho, también sabemos, gracias al mismo Latour y a otros historiadores de la ciencia como Isabelle Stengers o Michel Serres, que uno de los grandes problemas a los que se enfrenta el pensamiento científico es su escisión en disciplinas herméticas. Nace así la fractura entre las ciencias naturales y las ciencias sociales. Entre estudios tecnológicos y las artes. Si estudiamos la historia de la epistemología occidental nos encontraremos con una genealogía marcada por cortes, escisiones y campos que concurren para legitimar su capacidad para entender el mundo.

El filósofo Timothy Morton busca el origen de lo que él ha denominado «la gran amputación» (Morton, 2017), ese momento traumático en el que, para constituirse como humanos, nuestros antepasados tuvieron que negar los colectivos de los que eran parte. La naturaleza era lo que quedaba fuera del privilegio de ser humano. Según este autor, la revolución agrícola que dio paso al Neolítico fue el principio de la escisión humanos / no-humanos, con la domesticación de animales y plantas con las primeras formas de acumulación de bienes y objetos. Roberto Espósito, por su parte, acredita este hecho y ubica en la creación del derecho romano la materialización de esta amputación en un código que distingue las personas de las cosas (Espósito, 2015); quienes tienen agencia de los que obedecen órdenes; quienes tienen derechos de los que no. Estos dos eventos encuentran su cumbre en la modernidad. No ahondaremos en esto, puesto que en su obra Nunca fuimos modernos, Latour ya evidenció esta realidad exponiendo con solvencia como el proyecto moderno en el que se fundan nuestros marcos epistémicos es un proyecto de escisiones, fronteras, rupturas. La dicotomía entre humanos y no-humanos se consolidó. La ciencia de las cosas se separó de la ciencia de las personas. Lo objetivo de lo subjetivo. La presentación y la representación se fracturaron; las ciencias naturales se quedaron la primera; el pensamiento político, la segunda. Con esto, lo natural y lo social tuvieron que cortar vínculos; en una suerte de huida hacia adelante, los modernos lograron darle esquinazo al pensamiento premoderno. La magia debía quedar atrás; los hechos y los datos se auparon como espacio sin posible contestación o refutación. Sirviéndose de la controversia que se desató entre Boyle y Hobbes, Latour nos muestra cómo este cisma ayuda a vertebrar las epistemologías modernas. Por un lado, se estableció la ciencia de las cosas, las ciencias naturales; por otro, las ciencias de las personas o ciencias sociales. Por un lado, los objetos de la ciencia; por otro, los sujetos de derecho.

Este proceso, que ha tenido diferentes encarnaciones en la historia, no fue limpio ni estuvo exento de violencia. La modernidad implica una forma de violencia simbólica inusitada. Imponer el orden racional sobre las cosas, la dominación de los saberes científicos sobre los saberes tradicionales conllevaba una redistribución o apropiación de conocimientos. Nombrar, clasificar, asignar las palabras a las cosas. Como bien ha explicado la autora y activista Silvia Federici (2010), la normalización de los saberes fue una forma de arrebatar a las mujeres saberes ancestrales en el continente europeo, 0 a las comunidades indígenas en las colonias; en sus propias palabras: «El mundo debía ser desencantado para ser dominado» (Federici, 2010, pág. 243). Las epistemologías occidentales se impusieron sobre los saberes tradicionales, sobre las epistemologías andinas, amazónicas, indias, etc. Modernidad, colonialismo, heteropatriarcado. Un tridente que sirvió para normalizar cánones, establecer jerarquías de saberes, escindir formas de conocimiento, someter cuerpos y disciplinar a las personas.

Los modernos, armados de sus instrumentos científicos, categorías morales, carabelas, telescopios y pistolas se dedicaron a purificar los saberes por el mundo. Pensar y hacer, investigar y dibujar se volvieron actividades a todas luces irreconciliables. La ciencia y la política buscaron cauces, formas, espacios y jergas diferentes. Las academias se fracturaron en facultades. La luna se la quedaron las ciencias naturales. Nacieron los laboratorios para inscribir la realidad en superficies planas, en artículos científicos que nos explicaban cómo son las cosas. En palabras de Latour, se crearon los «móviles inmutables» (Latour, 1986b). Los agentes no humanos empezaban a tomar un papel preponderante en la producción de saberes científicos, así «dotados de sus nuevos poderes semióticos, [...] van a contribuir a una nueva forma de texto, el artículo de ciencia experimental, híbrido entre el estilo milenario de la exégesis bíblica (aplicada exclusivamente hasta ahora a las Escrituras y los clásicos) y el nuevo instrumento que produce nuevas inscripciones» (Latour, 1986b, pág. 47). La ciencia inscribe la realidad en documentos que después son usados como testigos de ella misma.

En este proceso de «depuración» de las ciencias naturales, estas perdieron su capacidad de reír, su humor (Stengers, 2000). Las ciencias dejaron de ser un espacio lúdico, creativo, dinámico y abierto para convertirse en un entorno marcado por la búsqueda 


\section{artnodes}

http://artnodes.uoc.edu

de la objetividad. El laboratorio de Boyle acabó con los salones de la ciencia. La magia, la intuición, el animismo, las estéticas o lo misterioso parecía no caber en los laboratorios. Por su parte, el ámbito de lo político se fue especializando en el arte de la representación. Diseñando instrumentos y máquinas que garantizan la representatividad, nacieron las soberanías, los sujetos, los parlamentos, las urnas. Estos nuevos órdenes sociales se hicieron con máquinas para medir lo social; máquinas de representación vicaria, como la estadística, el censo 0 el análisis social. Así nos encontramos con dos máquinas de ciencia escindidas, las naturales y las humanas; con sus mecanismos, instrumentos, aparatos, plataformas, espacios, técnicas. Sus sistemas de poder y validación. Sus verdades y sus métodos. Las artes, las estéticas y las poéticas, por su parte, se vieron relegadas a un discreto lugar, lejos de los laboratorios y de los parlamentos. La Luna era geología. Solo eso.

\section{Luna creciente}

A continuación vamos a explorar cómo lo expuesto anteriormente afecta a la investigación en diseño y, de forma más importante, dificulta su legitimación. Ahondaremos en cómo las fracturas y las divisiones entre áreas de conocimiento impactan en la propia naturaleza del diseño contemporáneo, en sus espacios de trabajo y aprendizaje, en las metodologías de investigación heredadas, sampleadas, inventadas, o en las cadenas de transmisión de saberes. Desde aquí sostenemos que la escisión y la amputación de saberes expuestos anteriormente han contribuido a invisibilizar el potencial epistemológico de ciertas prácticas de diseño contemporáneo. Ha contribuido a ocultar su capacidad de producir conocimiento. De ponerlo en práctica. De transferirlo. De evaluarlo. Y, de forma más dramática, de valorarlo.

Este hecho ayuda a entender por qué, en ocasiones, investigar en diseño se ha asimilado a imitar las formas y métodos de las ciencias naturales o incluso ha llevado a impostar las voces y formas propias de las ciencias sociales. Copiar sus metodologías cualitativas y cuantitativas, emular sus inscripciones o usar sus instrumentos de medición y validación. Esto ha ido en detrimento de valorar y desarrollar formas vernaculares de producir conocimiento, que se temía que eran de naturaleza más subjetiva o que estaban ocultas por la fuerza expresiva de las prácticas materiales sobre las que se articula el diseño. La intuición perdía su lugar, ocupado por el texto, el ensayo, la métrica y la medición. Por ello resulta cada vez más urgente, no tan solo en el ámbito del diseño, sino en la academia en general, explorar y crear metodologías inventivas de investigación capaces de unir éticas, estéticas y políticas (Lury y Wakeford, 2014; Back, 2013; Pink, 2015). Latour hace una llamada desde las ciencias que puede ser valiosa para el diseño: «Al enmendar la Constitución, seguimos creyendo en las ciencias, pero en vez de tomarlas en su objetividad, su verdad, su frialdad, su extraterritorialidad (cualidades que jamás tuvieron, sino por la recuperación arbitraria de la epistemología), las tomamos en lo que siempre tuvieron de más interesante: su audacia, su experimentación, su incertidumbre, su calor, su mezcla incongruente de híbridos, su loca capacidad para recomponer el lazo social» (Latour, 1986a, pág. 207). Las ciencias no son solo autoridad; son maleables, nos tienen que permitir jugar.

Las falsas dicotomías heredadas que han escindido teoría y práctica han impedido que se pueda pensar el diseño como un lugar donde legitimar procesos y métodos de investigación impuros, divertidos y bastardos. La partición entre el dicho y el hecho no permite ver con claridad esa mezcla incongruente de híbridos que reclama Latour. El desafío está en reorganizar el conocimiento, en unir y suturar culturas separadas del saber (Morin, 1999). Como bien explica Sennett en su libro El artesano, «la historia ha trazado falsas líneas divisorias entre práctica y teoría, técnica y expresión, artesano y artista, productor y usuario» (Sennett, 2009, pág. 23). La articulación de herramientas, cuerpos y materiales en culturas materiales que plantea Sennett resuena en las prácticas del diseño en las que la combinación de estos elementos, sus posibilidades y sus límites producen un rico campo de experimentación que acompaña a los procesos de investigación en diseño.

El legado de la escisión cartesiana entre cuerpo y mente ha hecho prácticamente imposible reconocer aquellas formas de producción de conocimiento que se encuentran lejos del brillante foco de la razón. La cabeza y la mano se alejan igual que se separan la intuición del intelecto. Por ello, el corte cartesiano ha sido puesto en duda desde diferentes áreas y disciplinas de saber. De forma notable, el neurocientífico Antonio Damasio expone cómo neuronalmente, emoción, sentimiento y regulación biológica desempeñan un papel en la razón humana. Narra cómo la toma de decisiones tanto personales como sociales se construye en un criterio conjunto que es regulado por todo el organismo, un organismo indisociable que interactúa continuamente con el ambiente. El aprendizaje, pues, se construye en esa relación entre cuerpo y mente, entre instinto y motivación, entre sentimiento y razón (Damasio, 1994, 2003).

Obviamente, aunque haya sido impuesta de forma violenta, la espistemología occidental no constituye el único marco epistémico del que podemos valernos para entender la realidad. Desde su crítica a la perspectiva única y en la revisión del perspectivismo amerindio y otras epistemologías, Viveiros de Castro pone en crisis algunos de los fundamentos del pensamiento moderno: «Educación, formación, conversión religiosa son procesos que, en nuestra tradición intelectual, se dan a nivel de espíritu (del intelecto, justamente). Los cambios en el plano del cuerpo no tenían, por lo menos hasta hace muy poco tiempo atrás, valor jurídico-metafísico discriminante. Digamos que, en el cuerpo de la modernidad, el cuerpo no tiene sentido» (Viveiros de Castro, 2008, pág. 66). Y es que, si retomamos la idea de investigación entendida como una forma de hacerse sensible, el cuerpo reclama 


\section{artnodes}

http://artnodes.uoc.edu

espacio. De aprendizaje. De movimiento. De experimentación. Espacio en el que tanto su lógica como su erótica no se den continuamente la espalda.

El diseño cohabita lugares de investigación práctica que heredan genealogías distintas con falsas líneas divisorias. Por un lado, está el laboratorio científico, ese lugar cerrado en el que el empirismo y la observación de fenómenos reproducidos artificialmente generan verdades (Latour, 1986a). Por otro, tenemos el aula, un lugar de disciplinamiento del cuerpo y de obediencia donde se generan relaciones de poder con jerarquías claras entre el que enseña y el que aprende (Foucault, 2009; Recalcati, 2016). Por otro, está el taller artesanal, ese espacio productivo donde el trabajo y la vida se entrecruzan en relaciones de autoridad y autonomía entre maestros, oficiales y aprendices (Sennett, 2009). Y también está el estudio del artista, donde la puerta se cierra para una puesta en escena privada en un gran ejercicio de subjetividad. Todos reordenan formas de entender las relaciones, las prioridades, las realidades, las posibilidades materiales, Ios aprendizajes, las transferencias y las producciones. Todos aportan pistas de cómo reconstruir nuevas redes de legitimación. La investigación en diseño busca su espacio, busca lugares en los que la cabeza y la mano formen parte del mismo escenario.

Las universidades, a su vez, han estado al servicio de centrar el conocimiento, de normalizarlo y transferirlo, de crearlo, y a la vez de evaluar y legitimar unos saberes y unas epistemologías concretas. Lejos de ser neutrales, también han contribuido a que otras fueran apartadas, ignoradas o devastadas. La investigación en diseño se compone de una serie inevitable de cruces. Cruces que necesitan de lugares donde acontecer, metodologías que las acompañen y una enseñanza afín. Con el auge de las universidades de diseño y la normalización que implica entrar en el marco del denominado «proceso de Bolonia» y la creación del Espacio Europeo de Educación Superior, la universidad ha asumido que es el lugar ideal para auspiciar la investigación en diseño. Aun así, hemos de buscar en otros contextos como los centros sociales, los entornos autogestionados, los laboratorios DIY, Ios fablabs o hackspaces, entornos ideales para que esta hibridación de saberes que comentábamos acontezca (Rowan, 2012; Rashof, 2016).

El diseño, ya lejos del paradigma de cumplir objetivos mercantilistas 0 encargos cerrados, abre sus puertas a mediar especulaciones, inventar ficciones, a abrir nuevas preguntas y a sostener dudas (Dunne y Raby, 2013). Investigar en diseño es dar rienda suelta a la curiosidad para así inventar nuevos mundos, esto requiere de marcos y formas de evaluación poco ortodoxas. El diseño contemporáneo ha dejado de ser esa disciplina cerrada basada en el oficio para abrirse a nuevas incertidumbres. Cuando se quiere investigar en diseño y no sobre él, no se trata de escribir sobre la producción gráfica de una serie de carteles o sobre la configuración de un espacio o sobre cómo se inventa un determinado artefacto, sino inventarlo, cacharrearlo, dar cuenta de sus tomas de decisiones, devolvérselo al mundo y averiguar qué ha sido de él. La investigación en diseño fuerza el tener que repensar sobre la propia naturaleza del oficio, de la profesión, de la institución, sobre el rol de la academia, las metodologías usadas y la evaluación de sus resultados. Sobre cuáles son los conocimientos tácitos que se practican y su necesidad y dificultad a la hora de transferirlos.

Veinte años después de las imposturas intelectuales de los físicos Alan Sokal y Jean Bricmont, en las que ya pusieron en evidencia la «falsa cientificidad» de los estudios sociales o humanidades, las verdades hipotéticamente demostrables y los razonamientos impecables y carentes de sentido (Sokal y Bricmont, 1998), se plantean otras formas de dar espacio a determinadas prácticas. Revisitar metodologías prestadas o inventadas es un ejercicio necesario para romper con la partición de la objetivación conocimientos y la subjetivación de expresividades. Trabajar en educar la mirada, en hacerse sensible, en generar saberes heterogéneos y salvajes conlleva ciertas revisiones en lo que exigimos como válido en un contexto de investigación. La purificación moderna nos ha conducido a clasificaciones obsoletas. Nos ha llevado a pensar el mundo a través de dicotomías imaginarias. Aun así nos empeñamos en separar lo político de lo científico, el conocimiento de la experiencia, los sujetos de los objetos, el centro de la periferia, lo mágico de lo funcional, el arte del mundo. Por eso damos la bienvenida a epistemologías salvajes, métodos experimentales y a las investigaciones inauditas.

\section{Conclusiones: Iuna Ilena}

Como hemos podido comprobar durante las páginas que preceden, la investigación en diseño seguramente tiene más que ver con lo que los modernos fueron dejando de lado que con los cánones, espacios y jergas que acabaron definiendo tanto las ciencias naturales como las sociales. Abriendo genealogías e hibridando esferas de conocimiento, el diseño es capaz de aglutinar pensamiento tecnológico con intuiciones artísticas. Preocupaciones sociales con una sensibilidad por los materiales y sus comportamientos. El diseño produce subjetividades y determina comportamientos políticos, a veces a su pesar. En ese sentido, la investigación en diseño se parece más a una luna dibujada en un cuaderno que a un laboratorio 0 a un parlamento. Si no es capaz de evidenciar híbridos, de concatenar teorías y prácticas, de permitir que lo sensible y lo sensual convivan con lo pragmático, la investigación en diseño lo va a tener muy difícil.

Por todo esto, seguramente, la investigación en diseño no surge de grandes hipótesis ni busca esclarecer formas de verdad, sino que ha aprendido del arte contemporáneo a lanzar preguntas que puede que no requieran de una respuesta... 0, por lo menos, de una respuesta cerrada (Dunne y Raby, 2013). El diseño perfila preguntas a través de la experimentación material y sensible (Boserman y Ricart, 2016). Así la investigación en diseño se torna un diálogo entre materiales, entre 


\section{artnodes}

http://artnodes.uoc.edu

planos y tornillos, entre patrones y telas, entre pantones y tipografías. La investigación en diseño produce artefactos que son objetos epistémicos, máquinas para hacer preguntas. Se experimenta inventando, que es lo que Stengers denomina «la invención del poder de conferir a las cosas el poder de conferir en el experimentador, el poder de hablar en su nombre» (Stengers, 2000, pág. 88). Las agencias saltan constantemente de bando. A veces son los objetos los que marcan la investigación; a veces son las palabras. Las intuiciones y las medidas. Los deseos y los encargos. La investigación en diseño se vehicula a través de artefactos, en ocasiones alucinantes, que sirven para enunciar hechos, para lanzar preguntas, para definir trayectorias (Martínez, Libow y Stager, 2013). En ese sentido se parece más a las ciencias experimentales que a las ciencias puras. Stengers señala que «se puede reconocer el paradigma teórico-experimental de la ciencia, no tan solo por la singularidad de su modo de fabricación de los hechos, sino también por su preocupación por el artefacto. Podríamos decir que cada hecho es un artefacto» ${ }^{2}$ (Stengers, 2000 , pág. 50). Materialidades, artefactos y sensibilidades. La investigación en diseño va tomando forma.

Si decíamos que las ciencias siempre han sabido producir los espacios y los mecanismos de legitimación de sus saberes, el laboratorio de Boyle, el congreso académico, la revisión entre pares, etc., el reto al que se enfrenta la investigación en diseño es el de crear espacios excéntricos en los que la estética no compita con la política, en los que la ética no riña con la erótica, o la intuición batalle con la razón (Boserman, Rocha, Rowan, 2015). En los que las divisiones artificiales del conocimiento se flexibilicen y den lugar a otros saberes y otras transferencias. Donde la inteligencia se pueda dar en los espacios de relación entre los sujetos y los objetos. Para ello convocamos aquellos espacios poco convencionales que han permitido que hacedores y hacedoras, piratas, activistas, subalternos, artistas, diseñadores y jaguares puedan coincidir y contribuir a producir epistemologías raras. Lugares que permiten que artistas y diseñadores generen ciencia, explorando nuevas subjetividades. Espacios que permiten sostener la incertidumbre y la provisionalidad. Sostener nuestras preguntas y explorar posibles respuestas materiales. Son estos espacios los que dan lugar a que las personas y las cosas puedan descentrarse y bailar coreografías inéditas. Es importante buscar los canales, las formas y los protocolos que permiten que estos espacios permeen las universidades y los espacios académicos. Que desborden las categorías existentes para generar nuevas ordenaciones de saberes y de conocimiento. Probablemente, estas serán más creativas, menos ortodoxas, menos cercados, menos fragmentarias, sin definiciones cerradas. Lo dice bien Stengers «cada definición excluye e incluye, justifica o cuestiona, crea o imposibilita un nuevo modelo» (Stengers, 2000, pág. 24).
Decíamos que en ocasiones las artes se han sentido huérfanas de lenguajes de investigación y se han visto obligadas a impostar voces para dotar a sus prácticas de legitimidad. Esto ha ido en detrimento de valorar y legitimar aquellos métodos de investigación vernaculares con los que ya se viene investigando en diseño. No podemos olvidar el poder del diagrama (Burke, 2014), las ficciones materiales (Fachetti, 2015) o el cacharreo como métodos de investigación que conllevan formas de inscripción, producen espacialidades y sistemas de verificación propios. Son métodos híbridos que pueden recombinarse y ensamblarse de forma exótica. Así la investigación en arte y diseño no pretende crear una nueva disciplina o marco normativo, más bien lo que se produce es una sensibilidad, un conjunto de formas de ver y sentir. Para concluir, recuperando la propuesta de Isabelle Stengers, nos gustaría creer que la investigación en diseño es un lugar ideal para recuperar la risa. Para que tenga lugar lo lúdico, lo inusitado, lo fabuloso. Para de forma desenfadada pero rigurosa poder explorar epistemologías excéntricas que nos ayuden a visibilizar nuevas relaciones entre objetos y sujetos. Para que la inventiva, la ingeniería, el cacharreo, la especulación y la diagramática puedan concatenarse de formas raras para producir nuevas formas de ver el mundo. De sentirlo. De vivirlo $y$, claro, de asumir que hacer diseño es atreverse a inventar mundos.

\section{Bibliografía}

BACK, L. (ed.) (2013). Live Methods. Londres: Blackwell.

BOSERMAN, C.; RICART, D. (2016). «Metodologías de investigación materializadas. Entre maquetas, tostadoras, diagramas, rampas y cabinas». INMATERIAL. Diseño, Arte y Sociedad. Vol. 1, n. ${ }^{0} 1$ (1). <https://www.inmaterialdesign.com/index.php/mag/article/ view/10>

BOSERMAN, C.; ROCHA, J.; ROWAN, J. (2015). «La materia contraataca: una tentativa objetológica». Obra digital. N. ${ }^{\circ}$ 9. <http:// revistesdigitals.uvic.cat/index.php/obradigital/article/view/66>

BURKE, A. (2014). «Considering the Diagram and Design Research». Lusofona Journal of Architecture and Education. N. ${ }^{0} 11$, págs.. 345 / 355.

DAMÁSIO, A. (2013). En busca de Spinoza: neurobiología de la emoción y los sentimientos. Barcelona: Booket.

DAMASIO, A. (1994). El error de Descartes: la emoción, la razón y el cerebro humano. Barcelona: Booket.

DUNNE, A.; RABY, F. (2013). Speculative Everything: Design, Fiction, and Social Dreaming. Cambridge, Massachusetts; Londres: The MIT Press.

ESPÓSITO, R. (2015). Persons and Things - From the Body's Point of View (1. a edición). Cambridge ; Malden, MA: Polity Press.

2. Aquí Stengers hace un juego de palabras difícil de traducir, en el que vincula hecho y arte: «We could say that every fact is here an artifact, a "fact of art"». 


\section{artnodes}

http://artnodes.uoc.edu

FACCHETTI, A. (2015). «Towards a political dimension of speculative design». [Fecha de consulta: 31/08/17] <https://www.academia. edu/23189960/Towards_a_political_dimension_of_speculative_design >

FARÍAS, I.; WILKIE. A. (eds.) (2015). Studio Studies: Operations, Topologies \& Displacements. Londres: Routledge.

FEDERICI, S. (2010). Caliban y la bruja. Madrid: Traficantes de Sueños.

FOUCAULT, M. (2009). Las palabras y las cosas. Madrid: Siglo XXI.

FRAYLING, C. (1994). «Research in Art and Design». Royal College of Art Research Papers. N. ${ }^{0}$ 1-01.

LATOUR, B. (1986a). Nunca fuimos modernos Ensayo de antropología simétrica. Madrid: Siglo XXI de España.

LATOUR, B. (1986b). «Visualization and cognition: thinking with eyes». En: Knowledge and Society - Studies in the Sociology of Culture Past and Present.

LATOUR, B. (2000). COGITAMUS Seis cartas sobre las humanidades científicas. Madrid: Paidós.

LURY, C.; WAKEFORD, N. (ed.). (2014). Inventive Methods. Londres: Routledge

MARKUSSEN, T.; STEINO (2012). «Design Research between Design and Research». Designskolen Kolding. <https://www.designskolenkolding.dk/pure/publication/1d1850b8-528f-471d-8175c22650a04647>

MARTíNEZ, S.; LIBOW G; STAGER, S. (2013). Invent To Learn: Making, Tinkering, and Engineering in the Classroom. Torrance, California: Constructing Modern Knowledge Press.
MORIN, E. (2000). La mente bien ordenada : repensar la reforma, reformar el pensamiento. Barcelona: Seix Barral.

MORTON, T. (2017). Humankind: Solidarity with Non-Human People. Brooklyn: Verso.

PINK, S. (2015). Doing Sensory Ethnography. Londres: Sage

RASHOF, S. (2016). «Designing Place - Topologies of Maker Labs». Londres: Goldsmiths, University of London.

RECALCATI, M. (2016). La hora de clase: por una erótica de la enseñanza. Barcelona : Anagrama.

RHEINBERGER, H. (2010). An Epistemology of the Concrete: TwentiethCentury Histories of Life. Experimental Futures. Durham, N. C.: Duke University Press.

ROWAN, J. (2012). «Fabricando Conocimiento/ Protocolos y metodologías de investigación en las comunidades de fabricación digital». $<$ https://gridspinoza.net/ca/node/196>

SENNETT, R. (2009). El artesano. Barcelona: Anagrama.

SOKAL, A. D.; BRICMONT, J. (1998). Intellectual Impostures: Postmodern Philosophers' Abuse of Science. Barcelona [etc.]: Profile Books.

STENGERS, I. (2000). Invention Of Modern Science. Minneapolis: University Of Minnesota Press.

SÁNCHEZ CRIADO., T.; RODRÍGUEZ-GIRALT, I. (2016). «Caring through Design?: En torno a la silla and the 'Joint Problem-Making' of Technical Aids». En: Care and Design: Bodies, Buildings, Cities. Bates, Kullman y Imrie (eds.). Wiley-Blackwell. Págs. 198-218.

VIVEIROS DE CASTRO, E. (2008). La mirada del jaguar. Introducción al perspectivismo amerindio. Río de Janeiro: Azougue.

\section{Cita recomendada}

ROWAN, Jaron; CAMPS, Marta (2017). «Investigación en diseño: suturando cuerpos, cacharros, epistemologías y lunas». En: Irma VILÀ y Pau ALSINA (coords.). «Arte e investigación». Artnodes. N. ${ }^{0} 20$, págs. 1-9. UOC [Fecha de consulta: dd/mm/aa] $<$ http://dx.doi.org/10.7238/a.v0i20.3133>

Este artículo está sujeto -si no se indica lo contrario- a una licencia de Reconocimiento 3.0 España de Creative Commons. Puede copiarlos, distribuirlos, comunicarlos públicamente, hacer obras derivadas y usos comerciales siempre que reconozca los créditos de las obras (autoría, nombre de la revista, institución editora) de la manera especificada por los autores o por la revista. La licencia completa se puede consultar en http://creativecommons.org/licenses/by/3.0/es/deed.es. 


\section{artnodes}

http://artnodes.uoc.edu

\section{CV}

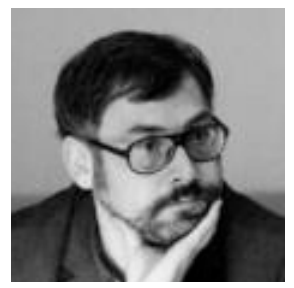

\section{Jaron Rowan}

BAU, Centro Universitario de Diseño de Barcelona

jaron.rowan@baued.es

Unidad de Investigación y Doctorado

c/ Pujades, 118

08005 Barcelona

Jaron Rowan es investigador cultural. Actualmente combina la investigación con la escritura y la docencia.

Es coordinador de la Unidad de Investigación y Doctorado de BAU, Centro Universitario de Diseño en Barcelona, donde también imparte la asignatura Economía, empresa y diseño. Es miembro de la línea de investigación Objetologías/GREDITS (<http://objetologias.tumblr. $\mathrm{com} />$ ). Durante varios años ha sido tutor y uno de los lectores del curso MA in Culture Industry (<https://www.gold.ac.uk/pg/ma-cultureindustry/>) de Goldsmiths University; además, ha impartido cursos y talleres en torno a asuntos relacionados con la economía de la cultura, las políticas culturales o el trabajo en cultura en numerosas universidades e instituciones culturales. Ha sido uno de los miembros fundadores de YProductions (2003-2013) y durante varios años ha sido uno de los coordinadores del Fcforum (<https://fcforum.net/>).

Ha escrito el libro Emprendizajes en cultura (<https://www. traficantes.net/index.php/editorial/catalogo/utiles/emprendizajes_en_cultura>), publicado en 2010 con la editorial Traficantes de Sueños (<https://www.traficantes.net/>), en el que, desde una óptica crítica, realiza un análisis de los principales programas de promoción del emprendizaje que actualmente se están llevando a cabo en el Estado español y donde evalúa algunas de sus consecuencias. De forma más reciente ha colaborado en libros como Cultura libre digital (Icaria, 2012), La tragedia del copyright (Virus, 2013) y ha publicado Memes: inteligencia idiota, política rara y folclore digital (Capitán Swing, 2015) (<http://capitanswing.com/libros/memes/>) y Cultura libre de Estado (<https://www.traficantes.net/libros/cultura-libre-de-estado>) (Traficantes de Sueños, 2016). 


\section{artnodes}

http://artnodes.uoc.edu

\section{CV}

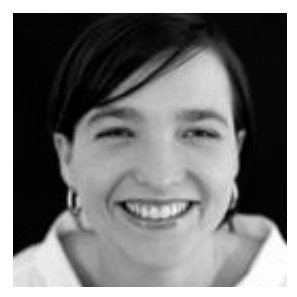

\section{Marta Camps}

BAU, Centro Universitario de Diseño de Barcelona

marta.camps@baued.es

Unidad de Investigación y Doctorado

c/ Pujades, 118

08005 Barcelona

Licenciada en Bellas Artes, máster en Teoría y Crítica de Arte y becada en el ENSBA de París. Es jefa de estudios y docente del Departamento de Arte del Grado en Diseño de BAU, Centro Universitario de Diseño en la que imparte Talleres de Dibujo, Color y Creatividad. Colabora en GREDITS en la línea de investigación innovación pedagógica en y mediante el diseño, en la que está desarrollando su tesis doctoral en el ámbito del aprendizaje de la creatividad. 